\title{
Compressed Synthetic Aperture Radar
}

\author{
Vishal M. Patel, Student Member, IEEE, Glenn R. Easley, Member, IEEE, Dennis M. Healy, Jr., and \\ Rama Chellappa, Fellow, IEEE
}

\begin{abstract}
In this paper, we introduce a new synthetic aperture radar (SAR) imaging modality which can provide a high-resolution map of the spatial distribution of targets and terrain using a significantly reduced number of needed transmitted and/or received electromagnetic waveforms. This new imaging scheme, requires no new hardware components and allows the aperture to be compressed. It also presents many new applications and advantages which include strong resistance to countermesasures and interception, imaging much wider swaths and reduced on-board storage requirements.
\end{abstract}

Index Terms-Synthetic aperture radar (SAR), compressed sensing (CS), compressive sensing.

\section{INTRODUCTION}

$\mathbf{S}$ YNTHETIC aperture radar (SAR) is a radar imaging technology that is capable of producing high-resolution images of the stationary surface targets and terrain. The main advantages of SAR are its ability to operate at night and in adverse weather conditions, hence overcoming limitations of both optical and infrared systems. The basic idea of SAR is as follows: as the radar moves along its path, it transmits pulses at microwave frequencies at an uniform pulse repetition interval (PRI) which is defined as 1/PRF, where PRF is the pulse repetition frequency. The reflected energy at any instant can be modeled as a convolution of the pulse waveform with the ground reflectivity function [1]-[3]. Each received pulse is preprocessed and passed on to an image formation processor. The image formation processor produces an image that is a two-dimensional mapping of the illuminated scene [4].

The two-dimensional image formed is interpreted in the dimensions of range and cross-range or azimuth. The range is the direction of signal propagation and the cross-range is the direction parallel to the flight path. Sometimes the range and the cross-range samples are referred to as the fast-time and the slow-time samples, respectively. The range resolution of a SAR image is directly related to the bandwidth of the transmitted signal and the cross-range is inversely proportional to the length

Manuscript received February 20, 2009; revised October 02, 2009. Current version published March 17, 2010. The associate editor coordinating the review of this manuscript and approving it for publication was Dr. Jared Tanner.

V. M. Patel, and R. Chellappa are with the Center for Automation Research, University of Maryland, College Park, MD 20742 USA (e-mail: pvishalm@umd.edu; rama@cfar.umd.edu).

G. R. Easley, is with the Department of Mathematics, University of Maryland, College Park, MD 20742 USA (e-mail: geasley@umd.edu).

D. M. Healy, Jr. (deceased) was with the Department of Mathematics, University of Maryland, College Park, MD 20742 USA.

Color versions of one or more of the figures in this paper are available online at http://ieeexplore.ieee.org.

Digital Object Identifier 10.1109/JSTSP.2009.2039181 of the antenna aperture. Therefore, high range resolution is achieved by transmitting wide bandwidth waveforms, and high cross-range resolution is achieved by coherently processing returns transmitted from a variety of positions along a flight path to emulate a large aperture.

There are four common modes of SAR: scan, stripmap, spotlight, and inverse SAR (ISAR). In this paper, we will mainly focus on the spotlight mode SAR and ISAR. In spotlight mode SAR, the radar sensor steers its antenna beam to continuously illuminate the terrain patch being imaged. It can provide higher resolution than the stripmap and scan mode SAR because it maps a scene at multiple viewing angles during a single pass [4]. In ISAR, the radar is stationary and the target is moving. The angular motion of the target with respect to the radar can be used to form an image of the target. Differential Doppler shifts of adjacent scatters on a target are observed and the target's reflectivity function is obtained through the Doppler frequency spectrum [5].

The standard methods for obtaining SAR images are basically based on using interpolation and the Fourier transform. One such method is known as the Polar Format Algorithm (PFA). In spotlight-mode SAR a collection of phase histories defines a set of samples in the Fourier space ( $k$-space) of the scene on an annular region. The PFA obtains the SAR image by appropriately interpolating these data from an annular region to a Cartesian grid and taking a two-dimensional inverse Fourier transform [4]. Another popular SAR image reconstruction method, based on the tomographic formulation of SAR [3], is the filtered backprojection (FBP) method [6]. Range migration and chirp scaling algorithms are also used for the spotlight mode SAR image reconstruction [4].

Since a SAR image is a map of the spatial distribution of the reflectivity function of stationary targets and terrain, many SAR images can be sparse or compressible in some representation such as those from wavelet or complex wavelet transform. ${ }^{1}$ The recently introduced theory of compressed sensing (CS) states that it is possible to recover such compressible images from a small number of random measurements provided that the undersampling results in noise like artifacts in the transform domain and an appropriate nonlinear recovery scheme is used [7], [8].

In this paper, we introduce a new SAR image formation algorithm empirically derived based on the theory of CS that reduces the number of transmitted and/or received waveforms. It will be demonstrated that if the SAR image is assumed to be compressible in some transform domain, then one can reconstruct a good

${ }^{1}$ SAR images are often characterized by the multiplicative noise known as speckle. Speckle makes the compressibility of the SAR images difficult. However, the underlying reflectivity map without the speckle has compressibility as good as some natural images. 
estimate of the reflectivity profile using this new image formation algorithm that relies on using a far fewer number of waveforms than the conventional systems do and requires no changes to a radar system hardware to work. It is also the case, that the radar community has considered similar concepts that we are presenting such as that provided in [9]. Yet our method enhances some of these suggestions and provides a framework along with general reconstruction techniques. By using concepts provided by CS theory we are able to propose an imaging system that should pave the way for many new applications that are highly desirable. In addition, not only do we demonstrate this concept of SAR imaging using CS theory with real data but we point out some of its valued benefits never before realized.

\section{A. Paper Organization}

In Section II, we describe previously radar related CS based imaging methods. The tomographic formulation of the spotlight mode SAR is given in Section III. In Section IV, we discuss the theory of CS and propose the SAR compressive sampling schemes in Section V. In Section VI, we show how the nonlinear recovery promoting sparsity can be used to estimate the SAR image from the undersampled phase histories. In Section VII, discussion of some of the possible applications of our proposed compressed synthetic aperture radar is given. In Section VIII, we show some of the experimental results, and present the concluding remarks in Section IX.

\section{PREVIOUS COMPRESSIVE RADAR RELATED WORK}

Inspired by the CS theory, more efficient schemes for sensing signals at much lower sampling rate than required by the traditional Nyquist sampling theorem have been proposed. This subNyquist acquisition framework is often known as the analog-toinformation (A2I) conversion [10]-[12]. Motivated by the CS theory, one such compressive radar imaging scheme based on A2I was proposed in [13]. It was argued that such a radar system can eliminate the need for the matched filter in the radar receiver and reduce the required receiver analog-to-digital conversion bandwidth [13]. A similar acquisition and imaging system for ground penetrating radar (GPR) was proposed in [14] and [15]. Instead of sampling the radar returns at the Nyquist rate, linear projections of the echo signals with random vectors were taken as measurements. It was shown that, using only a small subset of the measurements, sparser and sharper target images could be obtained compared to the standard backprojection method [14], [15]. In [16], a high-resolution radar was proposed based on CS by transmitting specially designed waveforms. A similar concept was also proposed in [17], where the theory of CS with random convolution was used by transmitting random noise like signals. Also, in [18], to reduce the amount of stored SAR data, a method based on CS theory was proposed. Recently, a method of imaging a scene of sparse targets from the perspective of scattering theory and CS has been proposed in [19].

Our approach in this paper is in contrast to some of the above mentioned compressive radar related algorithms that have only

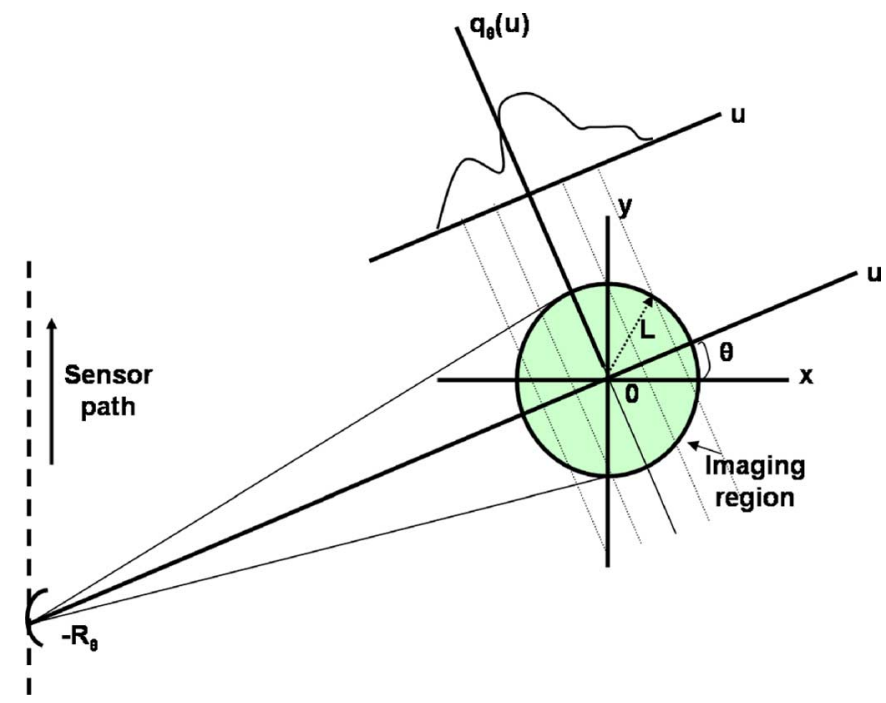

Fig. 1. Ground plane geometry in spotlight mode SAR.

considered using CS as part of the A2I conversion or transmitting specially designed waveforms. Note our method also requires no changes to a system's hardware to work unlike many other schemes that propose using CS theory for imaging.

\section{Spotlight Mode SAR Phase Histories}

In this section, we give a brief description of the tomographic formulation of the spotlight-mode SAR [3], [20]-[22]. The ground plane geometry in spotlight-mode SAR is shown in Fig. 1. The radar, which transverses the flight path, continuously points in the direction of a ground patch. At locations corresponding to each increments of $\theta$, high-bandwidth pulses are transmitted. The returns from the ground patch are received and processed to image the complex reflectivity profile $g(x, y)$.

In spotlight mode SAR, a linear FM (LFM) chirp signal is the most commonly used pulse, described mathematically as the real part of

$$
s(t)= \begin{cases}e^{j\left(\omega_{0} t+\alpha t^{2}\right)}, & |t| \leq \frac{T}{2} \\ 0, & \text { otherwise }\end{cases}
$$

where $\omega_{0}$ is the carrier frequency, $2 \alpha$ the chirp rate, and $T$ is the pulse length. The return from the scene patch of radius $L$ at the distance, $R_{\theta}$ where $\theta$ denotes the transmission angle, is then multiplied by the conjugate of a signal representing the return from the chosen reference point and low-pass filtered. Neglecting the residual video phase term and assuming $R_{\theta} \gg$ $L$, the demodulated signal is given by [20]

$$
\begin{aligned}
r_{\theta}(t)=\iint_{D} g(x, y) \exp \{ & -j \Omega(t) \\
& \times(x \cos (\theta)+y \sin (\theta))\} d x d y
\end{aligned}
$$

where $\Omega(t)=(2 / c)\left(\omega_{0}+2 \alpha\left(t-\tau_{0}\right)\right), c$ is the speed of light, $\tau_{0}=\left(2 R_{\theta}\right) /(c)$, and $D=\left\{(x, y) \mid x^{2}+y^{2} \leq L^{2}\right\}$. Equation (2) represents the projection slice at angle $\theta$ from the Fourier transform of the reflectivity field $g(x, y)$. Using the projection slice 
theorem, the signal $r_{\theta}(t)$ can also be identified as a band-pass filtered Fourier transform of the projections of the reflectivity field

$$
r_{\theta}(t)=\int_{-L}^{L} q_{\theta}(u) \exp \{-j \Omega(t) u\} d u
$$

where $q_{\theta}(u)$ is the projection of the reflectivity field $g(x, y)$ at angle $\theta$ described as

$$
q_{\theta}(u)=\iint_{D} \delta(u-x \cos (\theta)-y \sin (\theta)) g(x, y) d x d y .
$$

Taking advantage of a formulation of (2) that re-expresses $r_{\theta}(t)$ as $\left(\mathcal{A}_{\theta} g(x, y)\right)(t)$, where $\mathcal{A}_{\theta}$ is the continuous observation kernel, we can setup a matrix-based formulation of the imaging acquisition problem [21], [22]. Let $r_{\theta_{i}}\left(t_{j}\right)$ be the fasttime samples at the $i$ th observation angle $\theta_{i}$ at times $t_{j}$ of the continuous observation $r_{\theta_{i}}(t)$. Let $\mathbf{r}_{\theta_{i}}$ be the vector of these samples and $\mathbf{A}_{\theta_{i}}$ be a digital realization of the kernel $\mathcal{A}_{\theta_{i}}$, that is, the incorporation of all the linear image formation processes into a matrix expression and $\mathrm{g}$ be a lexicographically ordered vector of unknown sampled reflectivity field of length $\tilde{N}^{2}$. Then, one can write

$$
\left[\begin{array}{c}
\mathbf{r}_{\theta_{1}} \\
\mathbf{r}_{\theta_{2}} \\
\vdots \\
\mathbf{r}_{\theta_{P}}
\end{array}\right]=\left[\begin{array}{c}
\mathbf{A}_{\theta_{1}} \\
\mathbf{A}_{\theta_{2}} \\
\vdots \\
\mathbf{A}_{\theta_{P}}
\end{array}\right] \mathbf{g}
$$

or $\mathbf{r}=\mathbf{A g}$, where $\mathbf{r}=\left[\mathbf{r}_{\theta_{1}}, \ldots, \mathbf{r}_{\theta_{P}}\right]^{T}$, $\mathbf{A}=\left[\mathbf{A}_{\theta_{1}}, \ldots, \mathbf{A}_{\theta_{P}}\right]^{T}, P$ is the total number of slow-time samples used to form the image, and $\mathbf{A}$ is of size $P^{2} \times \tilde{N}^{2}$. For the sake of simplicity, we will assume that the number of phase histories and the number of samples per phase history are the same. The collection of phase histories $\mathbf{r}$ defines a set of samples in the Fourier space of the scene on an annular region, as shown in Fig. 2. Since this system relates the reflectivity profiles to the measurements directly, polar to rectangular sampling is not required [20]. Note, given the sampled phase histories $\mathbf{r}$, typically PFA is used to estimate the SAR image [4].

Assuming the presence of additive noise $\eta$, the SAR model can be expressed as $\mathbf{r}=\mathbf{A g}+\eta$. Assuming a similar SAR model, a regularized $\ell_{p}$-norm-based feature enhanced SAR image formation method has been used in [20], [21]. It is also possible to use the $\ell_{1}$-norm regularized reconstruction presented in [22] to obtain the sparse estimate of the reflectivity field.

\section{COMPRESSED SENSING}

The Shannon-Nyquist sampling theorem requires a signal to be sampled at a frequency of twice its bandwidth to be able to reconstruct it exactly. However, the recently introduced theory of compressed sensing enables the reconstruction of sparse or compressible signals from a small set of nonadaptive, linear measurements. If properly chosen, the number of measurements can be much smaller than the number of Nyquist rate samples

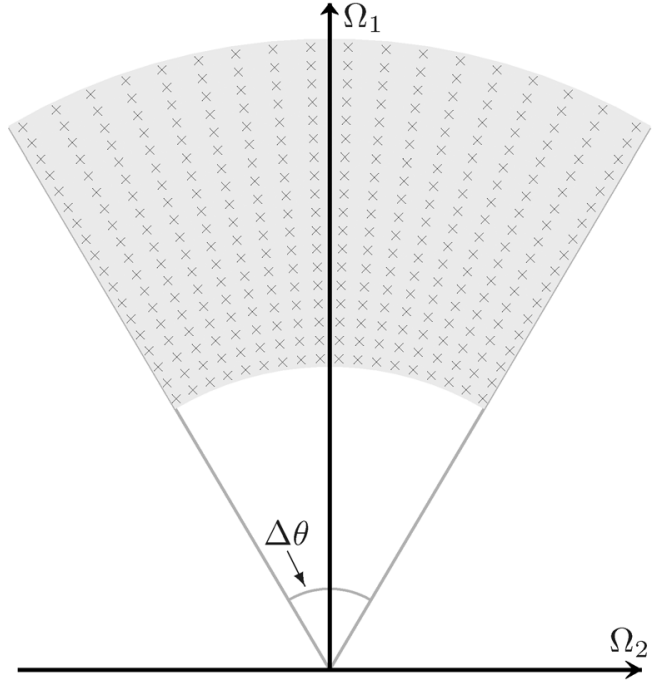

Fig. 2. Graphical representation of the phase histories on an annular region. Here, $\Omega_{1}, \Omega_{2}$, and $\Delta \theta$ denote spatial frequency in the range direction, spatial frequency in the cross-range direction, and the aperture angle, respectively.

[7], [8]. In this section, we give a brief introduction to the concept of compressed sensing.

Suppose $x \in \mathbb{C}^{N}$ is $K$-sparse in a basis or more generally a frame $\Psi$, so that $x=\Psi x_{0}$, with $\left\|x_{0}\right\|_{0}=K \ll N$, where $\|$ $x_{0} \|_{0}$ returns the number of nonzero elements of $x_{0}$. In the case when $x$ is compressible in $\Psi$, it can be well approximated by the best $K$-term representation. Consider an $M \times N$ measurement matrix $\Phi$ with $M<N$ and assume that $M$ linear measurements are made such that $y=\Phi x=\Phi \Psi x_{0}=\Theta x_{0}$. Having observed $y$ and knowing the matrix $\Theta$, the general problem is to recover $x_{0}$. Since $M<N$, this set of equations is underdetermined and has infinitely many solutions. To overcome this, the sparsest solution is usually sought, which can be done by solving the following optimization problem:

$$
\left(P_{0}\right) \arg \min _{x_{0}^{\prime}}\left\|x_{0}^{\prime}\right\|_{0} \text { subject to } y=\Theta x_{0}^{\prime} .
$$

Unfortunately, $\left(P_{0}\right)$ is NP-hard and is computationally difficult to solve. The approach taken in compressed sensing is to instead solve a relaxed version of $\left(P_{0}\right)$

$$
\left(P_{1}\right) \arg \min _{x_{0}^{\prime}}\left\|x_{0}^{\prime}\right\|_{1} \text { subject to } y=\Theta x_{0}^{\prime}
$$

where $\|x\|_{1}=\sum_{i}\left|\left(x_{i}\right)\right|$. The optimization problem $\left(P_{1}\right)$ is often known as Basis Pursuit (BP) [23] which can be solved by linear programming methods. It has been shown that under certain conditions on $\Theta$ and the sparsity of $x_{0}$, the solution to both $\left(P_{0}\right)$ and $\left(P_{1}\right)$ will coincide [24]. In the case when there are noisy observations of the following form:

$$
y=\Theta x_{0}+\eta
$$

with $\|\eta\|_{2} \leq \varepsilon$, Basis Pursuit De-Noising (BPDN) can be used to approximate the original image

$$
\left(P_{1}^{\varepsilon}\right) \arg \min _{x_{0}^{\prime}}\left\|x_{0}^{\prime}\right\|_{1} \text { subject to }\left\|y-\Theta x_{0}^{\prime}\right\| \leq \varepsilon .
$$


One sufficient condition for both $\left(P_{0}\right)$ and $\left(P_{1}\right)$ to have the same solution and for $\left(P_{1}^{\varepsilon}\right)$ to stably approximate the sparsest near-solution of (8), is known as the Restricted Isometry Property (RIP) [8], [25], [26]. A matrix $\Theta$ is said to satisfy the RIP of order $K$ with constants $\delta_{K} \in(0,1)$ if

$$
\left(1-\delta_{K}\right)\|v\|_{2}^{2} \leq\|\Theta v\|_{2}^{2} \leq\left(1+\delta_{K}\right)\|v\|_{2}^{2}
$$

for any $v$ such that $\|v\|_{0} \leq K$. The RIP essentially states that all subsets of $K$ columns taken from $\Theta$ are in fact nearly orthogonal. A related condition, known as incoherence, requires that the rows of $\Phi$ cannot sparsely represent the columns of $\Psi$ and vice versa [26]. Designing the matrix $\Phi$ such that the resulting sensing matrix $\Theta=\Phi \Psi$ has the RIP is a fundamental problem in CS. In fact, one can show that the RIP can be achieved with high probability by simply selecting $\Phi$ as a random matrix [26], [27].

In the case of SAR, the measurements may arise from a random selection of Fourier samples. In this case, the exact condition on the RIP depends on the incoherence between the Fourier basis and the sparsifying basis. In [28], Candès and Tao showed that when $\Phi$ is a partial Fourier matrix where $M$ rows of the $N \times N$ Fourier matrix are selected at random with renormalized columns, $\Psi$ is the identity matrix and if $K \leq C(M) /\left((\log (N))^{6}\right)$ for some constant $C>0$, then with high probability $\left(P_{1}\right)$ will recover $x_{0}$ exactly. ${ }^{2}$

Even though the RIP can be established for some matrices, in practice there is no computationally feasible way to check this property, as it is combinatorial in nature. However, there do exist related measures on the matrix $\Theta$ that can be used to prove the equivalence between $\left(P_{0}\right)$ and $\left(P_{1}\right)$ [30], [31]. One such measure on the matrix $\Theta$ is known as the mutual coherence. It is defined as follows:

$$
\mu=\mu(\Theta)=\max _{i \neq j} \frac{\left|\left\langle\vartheta_{i}, \vartheta_{j}\right\rangle\right|}{\left\|\vartheta_{i}\right\|_{2}\left\|\vartheta_{j}\right\|_{2}}
$$

where $\vartheta_{i}$ denotes the $i$ th column of the matrix $\Theta$. Equivalently, the mutual coherence can be viewed as the largest off-diagonal entry of the Gram matrix $G=\Theta^{T} \Theta$, where the columns of $\Theta$ are assumed to have been normalized [31]. We say $\Theta$ is incoherent if $\mu$ is small. It has been shown that the mutual coherence satisfies the following bound with the restricted isometry constants [32]

$$
\delta_{K} \leq(K-1) \mu .
$$

Finally, in certain cases, greedy algorithms such as orthogonal matching pursuit [33] can also be used to recover sparse images. In particular, a greedy algorithm known as, CoSaMP, is well supported by theoretical analysis and provides the same guarantees as some of the optimization based approaches [34].

${ }^{2}$ Recently, this result was improved in [29] and established $K \leq$ $C^{\prime}(M) /\left((\log (N))^{4}\right)$ for some constant $C^{\prime}>0$. It was also conjectured that $K \leq C^{\prime \prime}(M /(\log (N)))$ holds for some constant $C^{\prime \prime}>0$. This result can also be generalized to any sparsity basis $\Psi$ (see [27] for details).

\section{COMPRESSIVE SAMPLING FOR SAR}

The design of a CS undersampling scheme for SAR entails the selection of phase histories such that (11) is small. Some of the results about CS are based on the fact that the $k$-space samples are obtained randomly. However, sampling a truly random subset of the phase histories in SAR is usually impractical for existing hardware. In this section, we consider two compressed sensing $k$-space undersampling schemes for SAR. Since, the PRF essentially determines the slow-time sampling rate, our CS undersampling schemes are based on modifying the PRF of the radar. Implementation of such schemes is very simple and requires a minor change to the PRF scheduling of the radar.

Note that in practice the sparsity of the image will typically mean the percentage of transform coefficients needed to form an acceptable reconstruction. The acceptable reconstruction will depend on specific applications in mind such as whether it will be used for target identification or situational awareness. Thus, before such methods that we will suggest are fielded, systematic studies will be needed that will depend on their intended use.

\section{A. Random Slow-Time Undersampling}

As discussed earlier, as the sensor advances along its path, pulses are transmitted and received by the radar. The pulses are transmitted at every PRI $=(1) /(\mathrm{PRF})$. Undersampling methods based on sampling at regular intervals produce strong aliases. Random changes to the PRI can break up the periodicity of the aliasing artifacts and can convert strong aliases to random noise like artifacts [35], [36]. For this reason, instead of transmitting pulses with a regular PRI, we propose to transmit fewer pulses than traditional systems at random intervals. This amounts to undersampling the 2-D phase histories along the slow-time axis randomly.

\section{B. Jittered Slow-Time Undersampling}

Jittered undersampling is based on a regular undersampling which is perturbed slightly by random noise. The effect of jitter in one dimension was analyzed by Balakrishnan in [37]. He analyzed time jitter in which the $n$th sample is jittered by an amount $\zeta_{n}$ so that it occurs at time $n P_{T}+\zeta_{n}$, where $P_{T}$ is the sampling period. He reported that if the $\zeta_{n}$ are uncorrelated then the following happens: high frequencies are attenuated, the energy lost to the attenuation appears as uniform noise, and the basic structure of the spectrum does not change [35]-[37]. Jittered sampling in 2-D was generalized and applied in computer graphics in [35], [36]. Also, Hennenfent and Herrmann in [38] have successfully applied 2-D jittered undersampling in the context of $\mathrm{CS}$ for seismic data processing. Inspired by the properties of the jittered sampling, we propose to apply jittered undersampling in slow-time as well.

\section{Point Spread Function (PSF)}

To analyze the severity of artifacts introduced by these undersampling schemes, we adapt the notion of the point spread function (PSF) with regards to CS theory from [39]. Let $\mathbf{r}$ denote the collection of phase histories and $\widetilde{S}=\mathbf{R}_{\mathbf{S}} \mathbf{r}=\mathbf{R}_{\mathbf{S}} \mathbf{A g}=$ 
$\Phi \mathrm{g}$ represent the $k$-space measurements obtained by incorporating one of the slow-time undersampling schemes described above. Here, $\mathbf{R}_{\mathbf{S}}$ represents the restriction operator that selects the phase histories from the SAR model. Furthermore, we assume that $\mathbf{g}$ has a sparse representation (or is compressible) in some basis $\Psi$, so that $\mathrm{g}=\Psi v$. In this case, the sensing matrix $\Theta$ is given by $\Theta=\Phi \Psi$.

In [39], Lustig et al. proposed that the transform point spread function (TPSF) be used to measure the incoherence of a sampling scheme. It is defined as follows:

$$
\operatorname{TPSF}(m ; n)=\frac{\left\langle\Phi \Psi \mathbf{e}_{m}, \Phi \Psi \mathbf{e}_{n}\right\rangle}{\left\|\Phi \Psi \mathbf{e}_{m}\right\|_{2}\left\|\Phi \Psi \mathbf{e}_{n}\right\|_{2}}
$$

where $\mathbf{e}_{m}$ is the $m$ th vector of the natural basis having 1 at the $m$ th location and zeros elsewhere. Ideally, for $m \neq n$, the $\operatorname{TPSF}(m ; n)$ should be much smaller than 1 and should be noise like. This implies that the aliasing artifacts introduced by undersampling produces relatively small uncertainty in resolving $m$ th transform coefficient from the $n$th coefficient. In the case, when $\Psi$ is the identity matrix, we call the resulting TPSF simply the PSF. The maximum of the sidelobe-to-peak ratio (SPR) or the maximum off diagonal entry in TPSF, $\max _{m \neq n}|\operatorname{TPSF}(m ; n)|$ is used as a measure of severity of the aliasing artifacts [39]. Note that the SPR is also equal to the mutual coherence defined in (11).

\section{PSF Analysis}

In this section, we analyze the aliasing artifacts introduced from the slow-time undersampling schemes by the PSF. In the case, a sparsifying transform is used, TPSF can be studied to analyze the aliasing artifacts. We use the PSF of pure 2-D random sampling where samples are chosen randomly from a Cartesian grid, as a standard for comparison [39]. Fig. 3 shows the PSFs for random 2-D undersampling, random slow-time undersampling and jittered slow-time undersampling. The height of the red line measures the effect of the aliasing artifacts. The higher the line more severe the aliasing artifacts. It is clear from Fig. 3, that by undersampling the phase histories along the slow-time axis, we are mainly exploiting 1-D sparsity in 2-D. Therefore, the artifacts introduced by random or jittered slow-time undersampling are not as good as the one obtains with truly 2-D random $k$-space undersampling. Nevertheless, these aliasing artifacts can be removed using a nonlinear reconstruction technique promoting sparsity as suggested in [38], [39].

\section{SAR IMAGE RECONSTRUCTION}

Given the partial $k$-space measurements of the reflectivity map to be imaged, in this section, we show how the nonlinear recovery can be used to reconstruct the SAR image.

From the previous discussion, in the presence of additive measurement noise, we can write the partial $k$-space measurements as

$$
\begin{aligned}
\tilde{S} & =\mathbf{R}_{\mathbf{S}} \mathbf{A g}+\eta \\
& =\mathbf{R}_{\mathbf{S}} \mathbf{A} \Psi v+\eta=\Phi \Psi v+\eta=\Theta v+\eta
\end{aligned}
$$

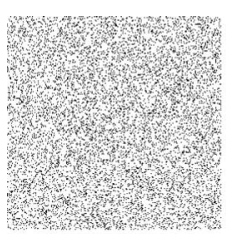

(a1)

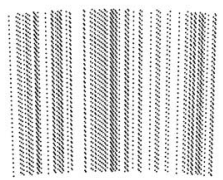

(b1)

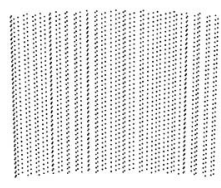

(c1)

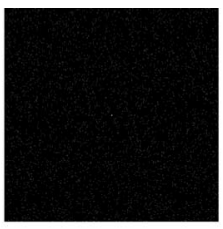

(a2)

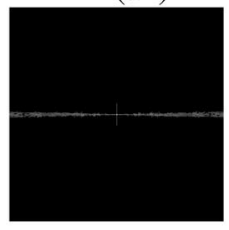

(b2)

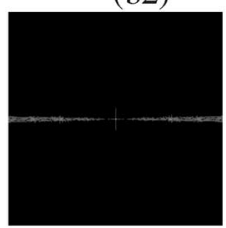

(c2)

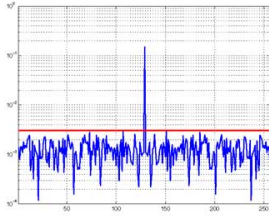

(a3)

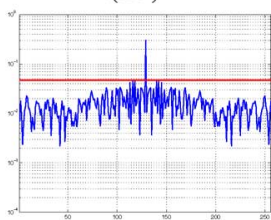

(b3)

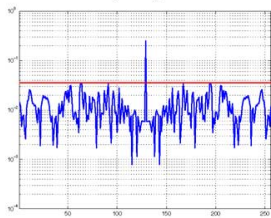

(c3)
Fig. 3. PSFs of various SAR undersampling schemes. (a1), (b1), (c1) Random 2-D points, Random slow-time undersampling, and Jittered slow-time undersampling, respectively. (a2), (b2), (c2) Image plots of the 2-D PSFs corresponding to (a1), (b1), and (c1), respectively, for all $m$ and a fixed $n$. (a3), (b3), (c3) 1-D slice through the 2-D PSFs in (a2), (b2), and (c2), respectively.

where $\eta$ is an arbitrary noise vector with $\|\eta\|_{2} \leq \varepsilon$, and $\mathbf{R}_{\mathbf{S}} \mathbf{A}$. Note the model in (14) may not be completely accurate as speckle is multiplicative, but this is a commonly used model for SAR (see [20] and [22]). The reflectivity map g can be estimated via $v$ by solving the following $\ell_{1}$ minimization problem

$$
v_{\text {rec }}=\arg \min _{v^{\prime}}\left\|v^{\prime}\right\|_{1} \text { s. t. }\left\|\tilde{S}-\Phi \Psi v^{\prime}\right\|_{2} \leq \varepsilon .
$$

It was shown in [31] that if $\|v\|_{0}<(1 / 4)((1 / \mu)+1)$ then the solution to (15) obeys

$$
\left\|v_{\mathrm{rec}}-v\right\|_{2}^{2} \leq \frac{4 \varepsilon^{2}}{1-\mu\left(4\|v\|_{0}-1\right)} .
$$

It is very difficult to prove any general claim that $\Theta$ satisfies a RIP or a mutual incoherence property for any particular sampling scheme. This remains an open problem. Thus, the best method for establishing mutual incoherence is to study the TPSF for a particular proposed sampling scheme given a particular scanning scenario. This presents no real obstacle in applications especially since many pre-calculations can be and are done before scanning begins. Note that, in the context of CS, the TPSF has successfully been used to characterize the incoherence of different sampling schemes arising in magnetic resonance [39] and in photo-acoustic tomography [40].

\section{A. Speckle}

Many coherent imaging modalities such as SAR, sonar, holography, and ultrasound often suffer form a multiplicative noise known as speckle. Speckle appears when objects illuminated by coherent radiation have surfaces that are rough compared with the imaging wavelength. It is caused by the constructive and destructive interference of the coherent returns 
scattered by small reflectors within each resolution cell [41], [42]. Speckle noise can make the detection and interpretation difficult for automated as well as human observers. In some cases, it maybe important to remove speckle to improve applications such as segmentation. To deal with speckle, instead of minimizing (15), we propose to minimize a modified problem

$$
\begin{aligned}
& v_{\text {rec }}=\arg \min _{v^{\prime}}\left\|v^{\prime}\right\|_{1}+\alpha \operatorname{TV}\left(\left|\Psi v^{\prime}\right|\right) \text { s.t. } \\
& \qquad \tilde{S}-\Phi \Psi v^{\prime} \|_{2} \leq \varepsilon
\end{aligned}
$$

for some user specified $\alpha \geq 0$ and TV is the Total Variation [45] defined as

$$
\operatorname{TV}(x)=\|\nabla(x)\|_{1} .
$$

By adding the TV constraint along with the $\ell_{1}$ constraint, we require the magnitude of the underlying complex SAR reflectivity field to be sparse in both the transform $\Psi$ and finite difference domains. The assumption that the underlying reflectivity field is piecewise smooth has been used before for image restoration under speckle noise [20], [43]-[45]. Note that no stability results have been proven for the minimization by the TV method. However, empirical experiments in [39] and [46] have shown that TV minimization provides the reconstruction as good as BP. Our experiments have shown that in practice (17) provides much better reconstruction in the presence of high speckle.

\section{APPLICATIONS}

The idea of transmitting waveforms at a nonuniform PRI for SAR has been suggested before [9]. This method, however, suffers from the smearing of the image in the cross-range dimension due to the randomness of the PRI. By solving the basis pursuit denoising problem (15), or (17), we are able to not only reconstruct the image as good as some of the traditional SAR reconstruction methods do from the full data but even at a lower sampling rate. Thus, our method of compressing the synthetic aperture offers many advantages.

\section{A. Counter-Countermeasure}

In many military applications of SAR, the user encounters scenarios where the adversary uses a transmitting radar to send out a signal within the band of the SAR system transmitter to confuse the SAR receiver. This process is called the electronic countermeasure (ECM) [47], [48]. The ECM causes the SAR system to receive and process erroneous information, which obscures targets or features of interest.

The ECM schemes used to jam a SAR system often relies on estimating the radar signal parameters such as PRI by exploiting the multiple transmissions of the signal at each synthetic aperture position. An elementary electronic counter-countermeasure (ECCM) is to jitter the PRF [47], [49]. Each outgoing pulse is either delayed or not depending on a sequence generated by a random number generator. A simpler implementation of changing the interpulse period is to drop pulses randomly. Hence, our compressive aperture method can offer strong countermeasures resistance [9], [47], [49].

\section{B. Reduction in Data}

In many SAR systems, radar data is directly stored on board and then transmitted to the ground in some reduced form. Our system has the potential to significantly reduce the amount of data to be stored and transmitted [2], [18].

Based on the CS theory, Bhattacharya et al. in [18], proposed a method of compressing the raw SAR data by using a simple encoder with a 2-D FFT and a random sampler. The decoding was done by using one of the CS recovery algorithms. Similarly, one can also encode the raw SAR data by using our compressed aperture method.

\section{Obtaining Wider Swaths}

In [50], Stoyle proposed a satellite imaging method that obtains an image of a wide swath of a planet's surface. $\mathrm{He}$ argued that by transmitting pulses at random PRIs, it is possible to image a much wider swath than possible by the conventional methods (see [50] for more details). Our method can also achieve the same task with an additional advantage of reduced data transmission.

\section{EXPERIMENTAL RESULTS}

In the following sections, we present results of our proposed CS SAR methods on synthetic and real SAR data.

\section{A. Phase Transition Diagrams}

We evaluate the performance of our compressed synthetic aperture radar methods by generating phase transition diagrams [51]. A phase transition diagram provides a way of checking $\ell_{0} / \ell_{1}$ equivalence, indicating how sparsity and indeterminancy affect the success of $\ell_{1}$ minimization [51], [52]. Let $\delta=(M / N)$ be a normalized measure of undersampling factor and $\rho=(K / M)$ be a normalized measure of sparsity. A plot of the pairing of the variables $\delta$ and $\rho$ describes a 2-D phase space $(\delta, \rho) \in[0,1]^{2}$. In the following experiments, the values of $\delta$ and $\rho$ ranged through 30 equispaced points in the interval $[0.03,1]$ and $N=900$. At each point on the grid, corresponding to a CS SAR model for certain values of $K, M$ and $N$ (in this case $N=900$ ), we recorded the mean number of coordinates at which original and reconstruction differed by more than $10^{-3}$, averaged over 30 independent realizations. In our approach, we employed a highly efficient algorithm that is suitable for large-scale applications known as the spectral projected gradient (SPGL1) algorithm [53], [54] for solving BP and BPDN problems such as (15).

In Fig. 4(a) and (b), we show the phase transition diagrams corresponding to the slow-time random and jittered undersampling, respectively, for the case when there is no complex white Gaussian measurement noise $(\eta=0)$ and $\Psi=I$ in (14). In Fig. 4(c) and (d), we show the phase transition diagrams for the slow-time random and jittered undersampling, respectively, for the case when the complex white Gaussian measurement noise $\eta$ has been added with signal-to-noise-ratio of $20 \mathrm{~dB}$ in (14). These plots indicate that the original target scene (image) can 


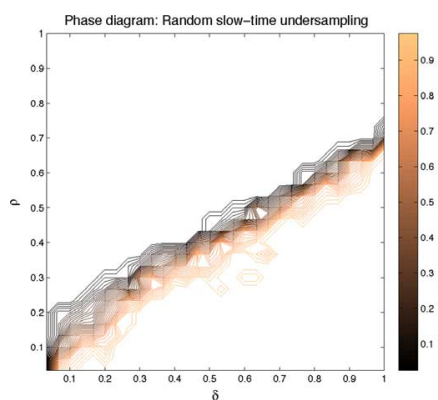

(a)

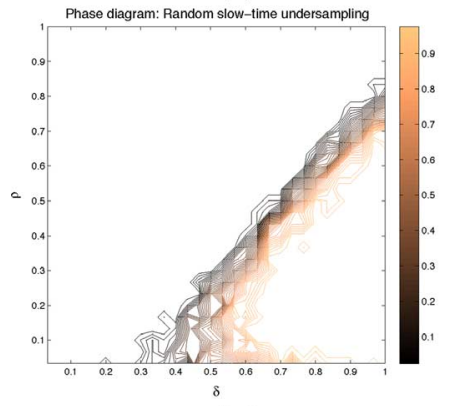

(c)

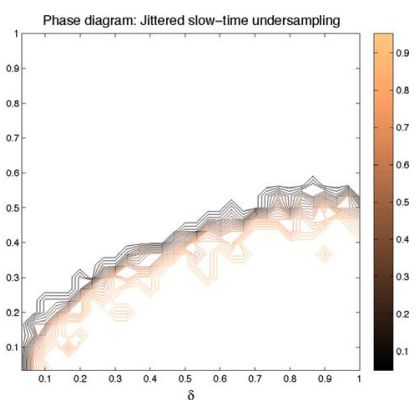

(b)

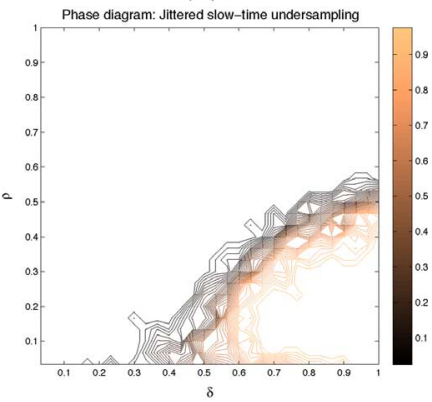

(d)
Fig. 4. Phase transition diagrams corresponding to Random slow-time undersampling (a) without noise, (c) with noise, and Jittered slow-time undersampling (b) without noise and (d) with noise.

be recovered well as long as it is sparse enough and enough measurements are taken.

\section{B. Reconstruction From the Compressive Measurements}

In this section, we demonstrate the performance and applicability of our compressive imaging algorithm on synthetic and real SAR data and compare it with the PFA. Note that the approximation underlying the PFA is generally poorer compared with the other algorithms. However, it is one of the most commonly used reconstruction methods for SAR which is why it is chosen for comparison.

In the first example, we used eleven point targets with unit magnitude to generate the phase histories. The control parameters used in our simulation are summarized in Table I. These determine all other variable parameters (see [4] for details). The range resolution is given by $\rho_{r}=(c / 2 B)=1.1 \mathrm{~m}$ and the cross-range resolution is given by $\rho_{\mathrm{cr}}=(\lambda) /(2 \Delta \theta)=1.1$ m. In Fig. 5(a), we show the generated phase histories from the eleven point targets. Fig. 5(b) shows the reconstruction of the point targets from the full simulated data using the PFA [4]. In Fig. 5(c), we show the phase histories obtained after applying $75 \%$ random slow-time undersampling ( $25 \%$ of data used). Fig. 5(d), shows the traditional reconstruction from the compressive measurements using the PFA. Fig. 5(e) shows the reconstructed image using our proposed method. It is clear from Fig. 5 that our method produces image from the compressive measurements as good as the PFA does from the full simulated data.

In the second experiment, we used ISAR data collected on a SAAB 9000 car using System Planning Corporation's Mark V

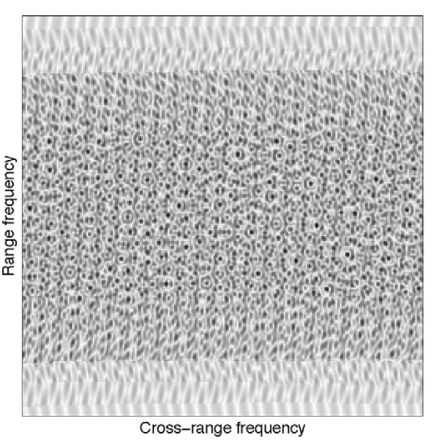

(a)

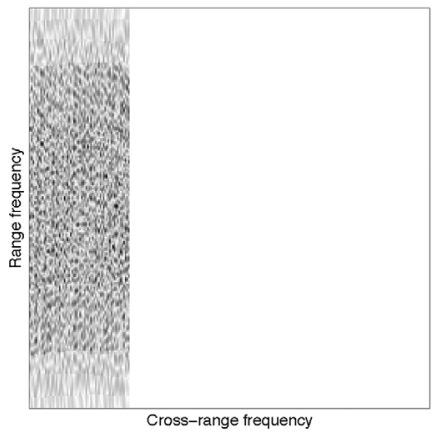

(c)

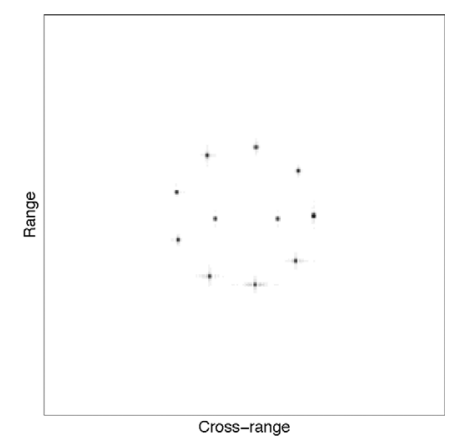

(b)

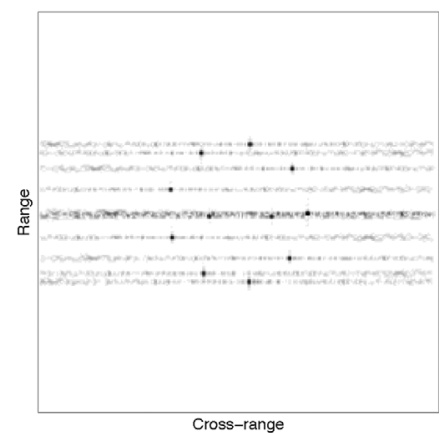

(d)

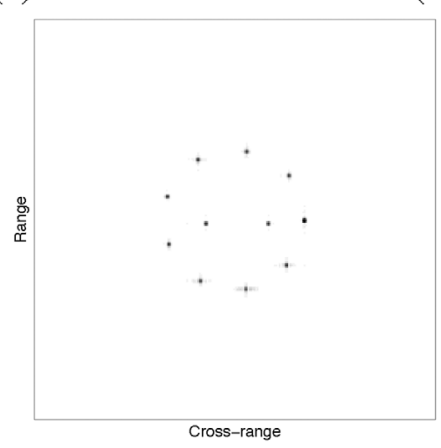

(e)

Fig. 5. Point targets example. (a) Simulated full data. (b) Traditional PFAbased reconstruction from the full simulated data. (c) Random slow-time undersampled phase histories (25\% of data used). (d) Reconstructed by the PFA from the compressive measurements. (e) Reconstructed image using our method.

TABLE I

PARAMETERS USED IN THE FIRST EXAMPLE

\begin{tabular}{|c|c|}
\hline Center frequency & $3.80 \times 10^{9} \mathrm{~Hz}$ \\
\hline Pulse width & $1 \times 10^{-6} \mathrm{sec}$ \\
\hline Bandwidth & $1.34 \times 10^{8} \mathrm{~Hz}$ \\
\hline Chirp rate & $1.34 \times 10^{14} \mathrm{~Hz} / \mathrm{sec}$ \\
\hline$\Delta \theta$ & $2.1^{\circ}$ \\
\hline
\end{tabular}

radar. ${ }^{3}$ We reconstructed the image after $60 \%$ jittered slow-time undersampling was applied to the data ( $40 \%$ of data used). As can be seen from Fig. 6, the reconstructed image from the compressed measurements, shown in Fig. 6(e), is identical to the one reconstructed from the full measurements, shown in Fig. 6(a). Fig. 6(d) shows how the traditional reconstruction fails to recover the ISAR image from the compressive measurements shown in Fig. 6(c).

\footnotetext{
${ }^{3}$ specifications of the radar can be found at www.sysplan.com/Radar.
} 


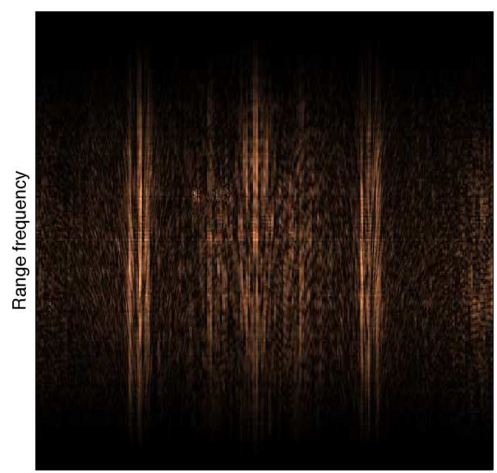

Cross-range frequency

(a)

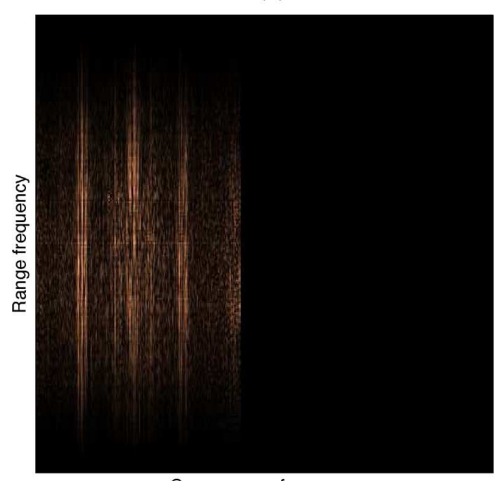

Cross-range frequency

(c)

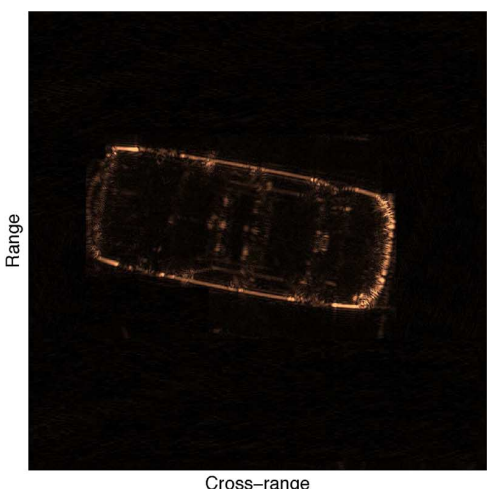

(b)

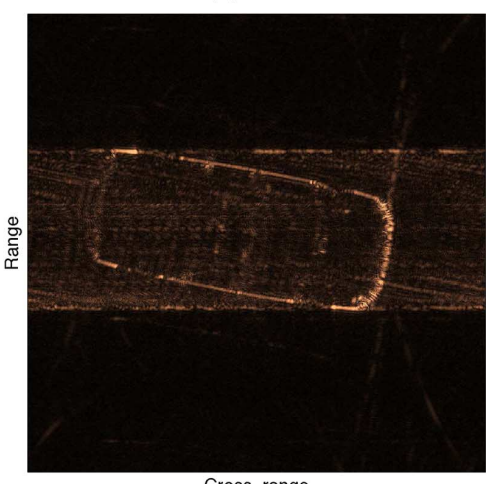

Cross-range

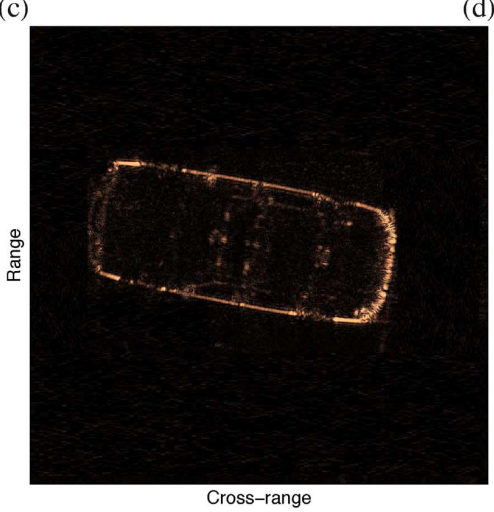

(e)

Fig. 6. SAAB 9000 car ISAR example. (a) Full measured data. (b) Traditional reconstruction from the full data. (c) Jittered slow-time undersampled phase histories (40\% of data used). (d) Traditional reconstruction from the compressive measurements in (c). (e) Reconstructed image using our proposed method.

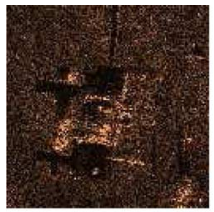

(a)

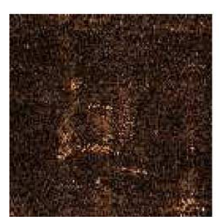

(d)

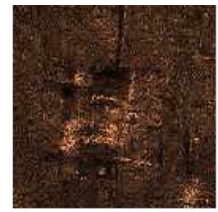

(b)

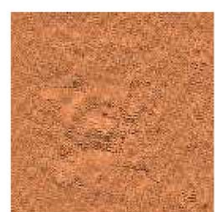

(e)

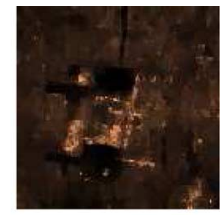

(c)

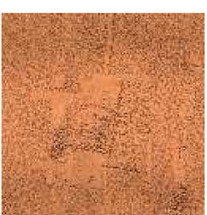

(f)
Fig. 7. MSTAR example. (50\% of data used) (a) Traditional reconstruction with the full data. (b) Reconstructed by solving (15). (c) Reconstructed by solving (17). (d) Reconstructed by using PFA. (e) The residual [i.e., (a)-(b)]. (f) The residual [i.e., (a)-(c)].
In the third experiment, we used a SAR image from the MSTAR public target database [55]. We simulated SAR phase histories using the acquisition method described in [21]. We used Daubechies 4 wavelet as a sparsifying transform for this experiment. The reconstruction from only $50 \%$ of the jittered slow-time undersampled data using the PFA, (15) and (17) is shown in Fig. 7(d), (b), and (c), respectively. In Fig. 7(e) and (f), we show how much speckle has been removed by solving (15) and (17), respectively. This experiment shows that it is possible to reconstruct and despeckle simultaneously from the compressive measurements. The value of $\alpha$ was chosen to be 3.8 after experimenting with different values for $\alpha$ and checking its performance.

In the final experiment, we used the ISAR dataset of a B-747 [5]. Fig. 8(a)-(c) shows the reconstructed image from the traditional method with full data, traditional method with partial data 


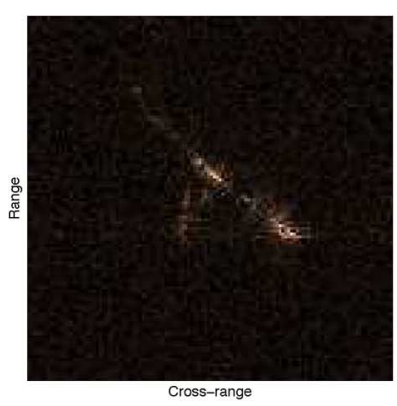

(a)

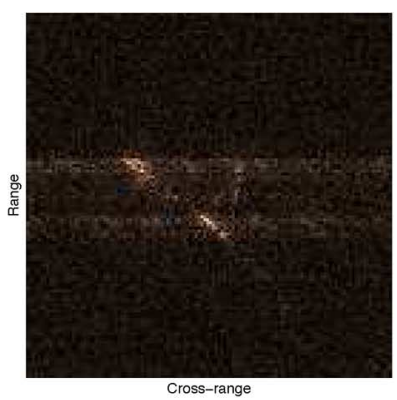

(b)

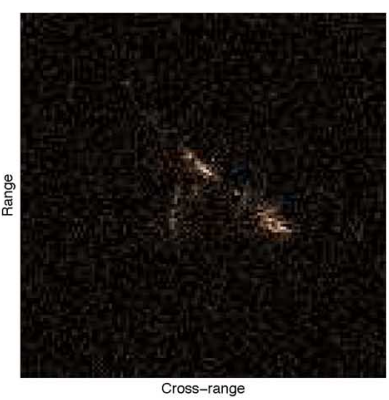

(c)

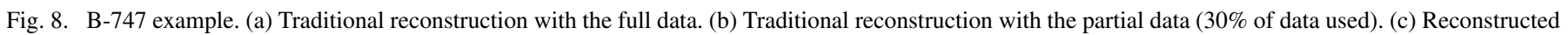
image using our proposed method.

and our method from the partial data, respectively. The reconstruction was done using $70 \%$ random slow-time undersampled phase histories (30\% of data used). As can be seen from Fig. 8 that our compressive imaging method provides a good reconstruction compared to the traditional method based on the full measurements.

Note that in our experiments the additive noise was either nonexistent or almost negligible. In the case when noise was nonexistent, $\varepsilon$ was chosen to be 0 . For the other cases, $\varepsilon$ was set to $10^{-2}$, which was a value we determined by trial and error.

It has been observed by many researchers [25], [39], [46], [56], that in practice, Fourier samples in the order of three to five times the number of sparse coefficients suffice for a good reconstruction. Our experiments also support this claim. In our experiments, we have noticed that taking more measurements generally improves the quality of reconstruction especially when the presence of speckle is high.

\section{Discussion AND CONCLUSION}

We have utilized CS theory and demonstrated that it is possible to compress the synthetic aperture for radar imaging. Most importantly, not only can our suggested undersampling be used in novel collection schemes to produce high-quality images but many new applications such as signals intelligence, imaging much wider swaths, and reduced storage constraints are possible because of it.

Further research is currently being done by the authors that includes many follow on ideas presented here. In particular, a more general model for SAR is being investigated where speckle is explicitly modeled. We are currently investigating this possibility of CS theory to deal directly with speckle noise. However, the $\ell_{1}$ minimization with the TV constraint seems to handle this but a more accurate formulation is still needed. We are also extending the application of our compressed SAR to ideas related to reducing the antenna size and the application in moving target indication using staggered PRIs.

\section{ACKNOWLEDGMENT}

Dennis Healy's contribution to this article was invaluable. He passed away only a few weeks before this manuscript was accepted for publication. We are honored to have worked with such a great man whose many contributions to the signal processing community have created such an important legacy. Furthermore, the authors would like to thank Jared Tanner and the anonymous reviewers for their many valuable comments and suggestions which significantly improved this paper.

\section{REFERENCES}

[1] M. Soumekh, Synthetic Aperture Radar Signal Processing With Matlab Algorithms. New York: Wiley, 1999.

[2] I. G. Cumming and F. H. Wong, Digital Processing of Synthetic Aperture Radar Data. Norwood, MA: Artech House, 2005.

[3] D. C. Munson, Jr., J. D. O'Brien, and W. K. Jenkins, "A tomographic formulation of spotlight-mode synthetic aperture radar," Proc. IEEE, vol. 71, no. 8, pp. 917-925, Aug. 1983.

[4] W. G. Carrara, R. S. Goodman, and R. M. Majewski, Spotlight Synthetic Aperture Radar: Signal Processing Algorithms. Norwood, MA: Artech House, 1995.

[5] V. C. Chen and H. Ling, Time-Frequency Transforms for Radar Imaging and Signal Analysis. Norwood, MA: Artech House, 2002.

[6] M. D. Desai and W. K. Jenkins, "Convolution backprojection image reconstruction for spotlight mode synthetic aperture radar," IEEE Trans. Image Process., vol. 1, no. 4, pp. 505-517, Oct. 1992.

[7] D. Donoho, "Compressed sensing," IEEE Trans. Inf. Theory, vol. 52, no. 4, pp. 1289-1306, Apr. 2006.

[8] E. Candès, J. Romberg, and T. Tao, "Robust uncertainty principles: Exact signal reconstruction from highly incomplete frequency information," IEEE Trans. Inf. Theory, vol. 52, no. 2, pp. 489-509, Feb. 2006.

[9] S. G. Mobley and M. W. Maier, "Synthetic aperture radar with a nonuniform pulse repetition interval," in Proc. SSST 27th Southeastern Symp. Syst. Theory (SSST'95), 1995, pp. 498-502.

[10] J. N. Laska, S. Kirolos, M. F. Duarte, T. S. Ragheb, R. G. Baraniuk, and Y. Massoud, "Theory and implementation of an analog-to-information converter using random demodulation," in Proc. IEEE Int. Symp. Circuits Syst. (ISCAS), May 27-30, 2007, pp. 1959-1962.

[11] J. Laska, S. Kirolos, Y. Massoud, R. Baraniuk, A. Gilbert, M. Iwen, and M. Strauss, "Random sampling for analog-to-information conversion of wideband signals," in Proc. IEEE CAS Workshop, Oct. 2006, pp. $119-122$.

[12] J. A. Tropp, J. N. Laska, M. F. Duarte, J. K. Romberg, and R. G. Baraniuk, "Beyond Nyquist: Efficient sampling of sparse bandlimited signals," IEEE Trans. Inf. Theory, vol. 56, no. 1, pp. 520-544, Jan. 2010.

[13] R. Baraniuk and P. Steeghs, "Compressive radar imaging," in Proc. 2007 IEEE Radar Conf., Apr. 2007, pp. 128-133.

[14] A. C. Gurbuz, J. H. McClellan, and W. R. Scott, "Compressive sensing for GPR imaging," in Proc. 41st Asilomar Conf. Signals, Syst. Comput. (ACSSC), Nov. 4-7, 2007, pp. 2223-2227.

[15] A. C. Gurbuz, J. H. McClellan, and W. R. Scott, "Compressive sensing for subsurface imaging using ground penetrating radar," Signal Process., vol. 89, no. 10, pp. 1959-1972, Oct. 2009.

[16] M. Herman and T. Strohmer, "High resolution radar via compressed sensing," IEEE Trans. Signal Process., vol. 57, no. 6, pp. 2275-2284, Jun. 2009. 
[17] J. Romberg, "Compressive sampling by random convolution," J. Imag. Sci., "," SIAM July 2008.

[18] S. Bhattacharya, T. Blumensath, B. Mulgrew, and M. Davis, "Fast encoding of synthetic aperture radar raw data using compressed sensing," in Proc. IEEE Workshop on SSP, Aug. 26-29, 2007, pp. 448-452.

[19] A. C. Fannjiang, P. Yan, and T. Strohmer, Compressed remote sensing of sparse objects Apr. 2009, submitted for publication.

[20] M. Çetin and W. C. Karl, "Feature-enhanced synthetic aperture radar image formation based on nonquadratic regularization," IEEE Trans. Image Process., vol. 10, no. 4, pp. 623-631, Apr. 2001.

[21] M. Çetin, "Feature-enhanced synthetic aperture radar imaging," Ph.D. dissertation, College of Eng., Boston Univ., Boston, MA, 2001.

[22] C. L. Logan, "An estimation-theoretic technique for motion-compensated synthetic-aperture array imaging," Ph.D. dissertation, Dept. of Elect. Eng. and Comput. Sci., Mass. Inst. of Technol., Cambridge, MA, Feb. 2000.

[23] S. Chen, D. Donoho, and M. Saunders, "Atomic decomposition by basis pursuit," SIAM J. Sci. Comp., vol. 20, no. 1, pp. 33-61, 1998.

[24] D. L. Donoho and X. Huo, "Uncertainty principle and ideal atomic decomposition," IEEE Trans. Inf. Theory, vol. 47, no. 7, pp. 2845-2862, Nov. 2001.

[25] E. Candès, J. Romberg, and T. Tao, "Satble signal recovery from incomplete and inaccurate measurements," Commun. Pure Appl. Math., vol. 59, pp. 1207-1223, 2006.

[26] R. Baraniuk, "Compressive sensing," IEEE Signal Process. Mag., vol. 24, no. 4, pp. 118-121, Jul. 2007.

[27] E. Candès, "Compressive sampling," in Proc. Int. Congr. Math., 2006, vol. 3, pp. 1433-1452.

[28] E. J. Candès and T. Tao, "Near-optimal signal recovery from random projections: Universal encoding strategies?," IEEE Trans. Inf. Theory, vol. 52, no. 12, pp. 5406-5425, Dec. 2006.

[29] M. Rudelson and R. Vershynin, "On sparse reconstruction from Fourier and Gaussian measurements," Commun. Pure Appl. Math., vol. 61, pp. 1025-1045, 2008.

[30] D. L. Donoho and M. Elad, "Optimally sparse representation in general (nonorthogonal) dictionaries via $\ell^{1}$ minimization," Proc. Nat. Acad. Sci., vol. 100, no. 5, pp. 2197-2202, Mar. 2003.

[31] D. L. Donoho, M. Elad, and V. N. Temlyakov, "Stable recovery of sparse overcomplete representations in the presence of noise," IEEE Trans. Inf. Theory, vol. 52, no. 1, pp. 6-18, Jan. 2006.

[32] H. Rauhut, K. Schnass, and P. Vandergheynst, "Compressed sensing and redundant dictionaries," IEEE Trans. Inf. Theory, vol. 54, no. 5, pp. 2210-2219, May 2008.

[33] J. A. Tropp and A. C. Gilbert, "Signal recovery from random measurements via orthogonal matching pursuit," IEEE Trans. Inf. Theory, vol. 53, no. 12, pp. 4655-4666, Dec. 2007.

[34] D. Needell and J. A. Tropp, "CoSaMP: Iterative signal recovery from incomplete and inaccurate samples," Appl. Comp. Harmonic Anal., vol. 26, pp. 301-321, 2008.

[35] R. L. Cook, "Stochastic sampling in computer graphics," ACM Trans. Graphics, vol. 5, no. 1, pp. 51-72, Apr. 1986.

[36] M. Dippe and E. Wold, "Antialiasing through stochastic sampling," SIGGRAPH, vol. 19, no. 3, pp. 69-78, Nov. 1985.

[37] A. V. Balakrishnan, "On the problem of time jitter in sampling," IRE Trans. Inf. Theory, vol. 8, no. 3, pp. 226-236, Apr. 1962.

[38] G. Hennenfent and F. J. Herrmann, "Simply denoise: Wavefield reconstruction via jittered undersampling," Geophysics, vol. 73, no. 3, pp. v19-v28, June 2008.

[39] M. Lustig, D. Donoho, and J. M. Pauly, "Sparse MRI: The application of compressed sensing for rapid MR imaging," Magn. Resonance Med., vol. 58, no. 6, pp. 1182-1195, Dec. 2007.

[40] J. Provost and F. Lesage, "The application of compressed sensing for photo-acoustic tomography," IEEE Trans. Med. Imag., vol. 28, no. 4, pp. 585-594, Apr. 2009.

[41] J. Goodman, "Some fundamental properties of speckle," J. Opt. Soc. Amer., vol. 66, pp. 1145-1150, Nov. 1976.

[42] C. V. Jakowatz, Jr., D. E. Wahl, P. H. Eichel, D. C. Ghiglia, and P. A. Thompson, Spotlight-Mode Synthetic Aperture Radar: A Signal Processing Approach. New York: Springer, 1996.

[43] M. Çetin, W. C. Karl, and A. S. Willsky, "Feature-preserving regularization method for complex-valued inverse problems with application to coherent imaging," Opt. Eng., vol. 54, no. 1, Jan. 2006, 017003.
[44] R. Lavarello, F. Kamalabadi, and W. D. O’Brien, Jr., “A regularized inverse approach to ultrasonic pulse-echo imaging," IEEE Trans. Med. Imag., vol. 25, no. 6, pp. 712-722, Jun. 2006.

[45] J. Shi and S. Osher, "A nonlinear inverse scale space method for a convex multiplicative noise model," Comput. and Appl. Math., Univ. of California, Los Angeles, Tech. Rep. 07-10, 2007.

[46] E. Candes and J. Romberg, "Signal recovery from random projections," in Proc. SPIE Comput. Imag. III, San Jose, CA, 2005, vol. 5674.

[47] W. W. Goj, Synthetic Aperture Radar and Electronic Warfare. Norwell, MA: Artech House, 1992.

[48] G. E. Stimson, Introduction to Airborne Radar, 2nd ed. Mendham, NJ: SciTech, 1998.

[49] R. J. Anderson, Security Engineering: A Guide to Building Dependable Distributed Systems. Indianapolis, IN: Wiley, 2008.

[50] P. N. Stoyle, "Synthetic aperture radar assembly and a method of creating a radar image of a planet surface using such an assembly," U.S. patent 5,051,749, Sep. 24, 1991.

[51] D. L. Donoho, "High-dimensional centrally symmetric polytopes with neighborliness proportional to dimension," Discrete and Comput. Geom., vol. 35, no. 4, pp. 617-652, 2006.

[52] J. D. Blanchard, C. Cartis, and J. Tanner, "The restricted isometry property and $\ell_{q}$-regularization: Phase transition for sparse approximation," submitted for publication.

[53] E. van den Berg and M. P. Friedlander, "Probing the Pareto frontier for basis pursuit solutions," SIAM J. Sci. Comp, vol. 31, no. 2, pp. 890-912, 2008.

[54] E. van den Berg and M. P. Friedlander, "SPGL1: A solver for large-scale sparse reconstruction." [Online]. Available: http://www.cs. ubc.ca/labs/scl/spgl1

[55] Center for Imaging Science Image Database [Online]. Available: http:// cis.jhu.edu/data.sets/MSTAR

[56] Y. Tsaig and D. L. Donoho, "Extensions of compressed sensing," Signal Process., vol. 86, no. 3, pp. 533-548, Mar. 2006.

[57] J. A. Fessler and B. P. Sutton, "Nonuniform fast Fourier transforms using min-max interpolation," IEEE Trans. Signal Process., vol. 51, no. 2, pp. 560-574, Feb. 2003.

[58] M. M. Bronstein, A. M. Bronstein, M. Zibulevsky, and H. Azhari, "Reconstruction in diffraction ultrasound tomography using nonuniform FFT," IEEE Trans. Med. Imag., vol. 21, no. 11, pp. 1395-1401, Nov. 2002.

[59] J. Walker, "Range-Doppler imaging of rotating objects," IEEE Trans. Aerosp. Electron. Syst., vol. AES-16, no. 1, pp. 23-52, Jan. 1980.

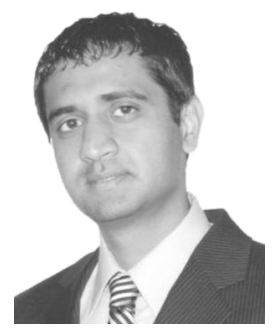

Vishal M. Patel (M'01) received the B.S. degree in electrical engineering and applied mathematics (with honors) and the M.S. degree in applied mathematics from North Carolina State University, Raleigh, in 2004 and 2005, respectively. He is currently pursuing the $\mathrm{Ph} . \mathrm{D}$. degree in electrical engineering at the University of Maryland, College Park.

His research interests include compressed sensing, radar imaging, pattern recognition, and biometrics.

Mr. Patel is a member of Eta Kappa Nu, Pi Mu Epsilon, Phi Beta Kappa, and a student member of

SIAM.

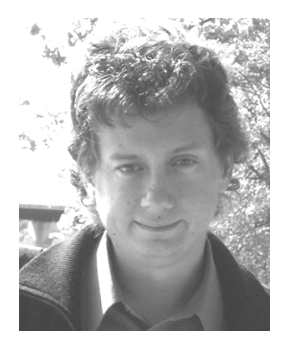

Glenn R. Easley (M'08) received the B.S. (with honors) and M.A. degrees in mathematics from the University of Maryland, College Park, in 1993 and 1996, respectively, and the Ph.D. degree in computational science and informatics from George Mason University, Fairfax, VA, in 2000.

Since 2000, he has been with System Planning Corporation working in signal and image processing. $\mathrm{He}$ has also been a Visiting Assistant Professor for the Norbert Wiener Center, University of Maryland, since 2005. His research interests include computational harmonic analysis, with special emphasis on wavelet theory and time-frequency analysis, synthetic aperture radar, deconvolution, and computer vision. 


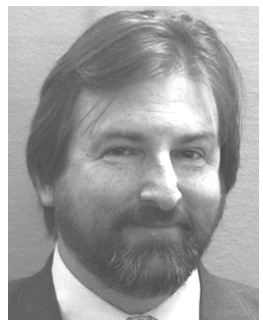

Dennis M. Healy, Jr. received the B.S. degrees in physics and mathematics and the Ph.D. degree in mathematics from the University of California at San Diego (UCSD), La Jolla, in 1980 and 1986, respectively.

He was a Professor of mathematics at the University of Maryland, College Park, as well as Program Manager for the Microsystems Technology Office at DARPA. Previously, he served as a Program Manager for DARPA's Applied and Computational Mathematics Program in the Defense Sciences Office and as Associate Processor at Dartmouth College, Hanover, NH, with joint appointments in the Departments of Mathematics and Computer Science. His research concerns applied computational mathematics in real-world settings including medical imaging, optical fiber communications, design and control of integrated sensor/processor systems, control of quantum systems, statistical pattern recognition, and fast nonabelian algorithms for data analysis.

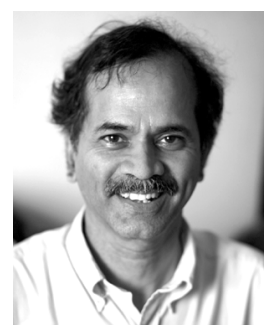

Rama Chellappa (F'92) received the B.E. (honors) degree from the University of Madras, Madras, India, in 1975, the M.E. (distinction) degree from Indian Institute of Science, Bangalore, in 1977, and the M.S.E.E. and Ph.D. degrees in electrical engineering from Purdue University, West Lafayette, IN, in 1978 and 1981, respectively.

Since 1991, he has been a Professor of Electrical Engineering and an affiliate Professor of Computer Science at University of Maryland, College Park. He is also affiliated with the Center for Automation Research (Director) and the Institute for Advanced Computer Studies (Permanent
Member). In 2005, he was named a Minta Martin Professor of Engineering. Prior to joining the University of Maryland, he was an Assistant (1981-1986) and Associate Professor (1986-1991) and Director of the Signal and Image Processing Institute (1988-1990) at the University of Southern California, Los Angeles. Over the last 28 years, he has published numerous book chapters and peer-reviewed journal and conference papers. He has coauthored and edited books on MRFs, face and gait recognition, and collected works on image processing and analysis. His current research interests are face and gait analysis, markerless motion capture, 3-D modeling from video, image and video-based recognition and exploitation. and hyper spectral processing

Prof. Chellappa served as an Associate Editor of four IEEE Transactions, as a Co-Editor-in-Chief of Graphical Models and Image Processing and as an Editor-in-Chief of the IEEE TRANSACTIONS ON PATTERN ANALYSIS AND MACHINE INTELLIGENCE. He served as a member of the IEEE Signal Processing Society Board of Governors and as its Vice President of Awards and Membership. He is serving a two-year term as the President of IEEE Biometrics Council. He has received several awards, including an NSF Presidential Young Investigator Award, four IBM Faculty Development Awards, an Excellence in Teaching Award from the School of Engineering at USC, two paper awards from the International Association of Pattern Recognition, Technical Achievement, and Meritorious Service Awards from the IEEE Signal Processing Society and the IEEE Computer Society. At the University of Maryland, he was elected as a Distinguished Faculty Research Fellow, as a Distinguished Scholar-Teacher, received the Outstanding Faculty Research Award from the College of Engineering, an Outstanding Innovator Award from the Office of Technology Commercialization and an Outstanding GEMSTONE Mentor Award. He is a Fellow of the International Association for Pattern Recognition and Optical Society of America. He has served as a General Technical Program Chair for several IEEE international and national conferences and workshops. He is a Golden Core Member of the IEEE Computer Society and serving a two-year term as a Distinguished Lecturer of the IEEE Signal Processing Society. 